\title{
Study of Machinery Hand Auto-control Technology based on PLC \\ Man $\mathrm{Li}^{1, \mathrm{a}}$ \\ ${ }^{1}$ Xi'an International University, Xi'an, Shaanxi, 710077 \\ a email
}

\section{Keywords: Machinery Hand, Auto-control Technology}

\begin{abstract}
A robot is a positioning control can be automated and can be reprogrammed to change the multifunctional machine, occupies an important position in the industrial automation production. Hydraulic drive system can be pneumatic drive, it may be electrical control and other methods. With sensor technology, pneumatic technology, the development of computer technology, computer-based technology-based control technology developed rapidly, including pneumatic technology to economic, inexpensive, sensitive, etc., and become one of the hotspots. This article describes a model of the robot hand control system based on a dedicated computer -PLC (Programmable logic Controller, called PLC).
\end{abstract}

\section{Introduction}

Driving system robot has pneumatic drive, hydraulic drive, electric drive and mechanical drive. Pneumatic technology is widely used compressed air as medium, with quick action, stable and reliable, simple structure, light, small, energy-saving, long working life characteristics, especially easy to control, easy maintenance, no environmental pollution case, So often pneumatic technology as the preferred robot drive system. Compared with other pneumatic manipulator control robot, with no pollution, strong anti-interference, low cost, simple structure, power and high volume. In the machinery industry, more and more automated equipment used in the robot, the main hydraulic control and pressure control in two ways. Wherein the pneumatic manipulator with its inexhaustible, with the endless source of gas, and lower production costs by the user favorite, National research on pneumatic manipulator more and more attention, has become an important meet production needs practical technology.

Industrial robot (hereinafter referred to as the robot) is a new technology in the field of modern automatic control appears, has become a modern manufacturing system is an important part. The rapid development of the robot is due to the positive role it has increasingly been recognized: First, it can partly replace the manual operation; secondly, it can in accordance with the requirements of the production process, to follow certain procedures, time and location to complete delivery and unloading of the workpiece; Third, it can carry out operations necessary equipment Fai pick and assembly. Thus, it can greatly improve the working conditions of workers, significantly improve labor productivity, accelerate the pace of industrial production mechanization and automation. Thus, the robot attention in the advanced industrial countries, and put a lot of manpower and resources to research and applications, especially in high temperature, high pressure, dust, noise and radioactive properties and polluting, more widely used.

With the rapid development of modern industrial production, the robot in the world can be widely applied. Thus the manipulator control requirements are also getting higher and higher, if the traditional relay control scheme to control system components will inevitably result in more complex wiring, poor stability, high failure rate, caused much inconvenience to the industrial production. If the high cost performance PLC programmable logic controller (PLC based advantages described in the next section) to address these issues to design their control system that allows high reliability of the system, low failure rate, easy maintenance, made good work results.

\section{Robot Working Environment}

Based on the above analysis, the robot requires a high temperature to withstand the conditions under 
heavy load, the bar can achieve fast, accurate handling. This replaces the manual operation, greatly improved working conditions for workers, significantly improve labor productivity.

Should have enough grip (clamping force) in determining the grip of the finger, in addition to considering the weight of the work, it must also consider the inertia forces and vibration during operation or transmission generated to ensure that the work will not have a loose or fall off.

Fingers should have a certain angle of opening and closing two fingers open and close the angle of the two extreme positions sandwiched finger Jian called angle closure. Finger Jian angle closure should ensure that the workpiece can successfully enter or off Jian. If the workpiece clamping different diameters, the maximum diameter of the workpiece should be considered. Movable finger for closing only Jian amplitude requirements.

Should ensure accurate positioning of the workpiece so that the workpiece is clamped fingers and maintain accurate relative position must be based on the grasped workpiece shape, select the shape of the fingers. Such as a cylindrical workpiece preclude fingers with "V" shaped face, so that self-centering.

Should have sufficient strength and stiffness of the fingers except the workpiece is held by a reaction force, but also affected the movement of the robot generated during the inertia forces and vibration, require sufficient strength and rigidity against bending or breaking, but we should try to make the structure simple and compact, light weight, center of gravity and the hand on the wrist of the axis of rotation, so that the smallest wrist twisting moment better.

Should be considered to be crawled objects in claim 1) should be based on the shape of the fingers crawl shape workpiece shape and design. As the workpiece is cylindrical, V-shaped finger is used, spherical arc-shaped workpieces finger three fingers, square planar material with the fingers, filament workpiece with a sharp hook shaped finger or a fine-toothed jaw fingers. In conclusion it should be selected according to the workpiece shape finger shape. 2) Crawl portion size is the same as far as possible, if after processing, varying in size, the finger should be able to adapt to changes in the size of the request, as otherwise allowed to crawl the site. For high surface quality requirements, try to avoid the high-quality surface or on a finger plus soft Sook sheet (such as rubber, foam, asbestos gaskets, etc.) to prevent damage to the workpiece clamping crawling. 3) If the number of crawl crawling finger with a pair plurality of workpieces, in order to loosen the individual workpiece or off phenomenon does not occur, increase the elasticity of the lining of heat, such as rubber, foam fingers and so on, for a long workpieces can be dual or more fingers crawl.

You should consider the versatility finger finger is a dedicated member of the strong, to meet the requirements of different shapes and sizes of small quantities of many varieties of the workpiece can be made modular fingers. For this finger requiring a simple structure, easy installation and maintenance, easy to replace.

\section{Robot PLC Control System Design}

Programmable controller consists of the CPU unit, an input unit, an output unit, extension unit and programmer composition and other components.

CPU module consists of a microprocessor (CPU chip) and memory composed of the programmable controller system, CPU module is equivalent to the human brain and heart, it continues to capture the input signal, the user program execution, refresh the system output, memory for storing programs and data.

Input (Input) module and output (Output) module is referred to as the I / O modules, which is the system's eyes, ears, hands, feet, is an external site link bridge and CPU module. One is the input signal from the button, selector switches, digital DIP switches, limit Guan Jian, an amount close to the Jian Guan Jian Guan, photoelectric switches, relays and other pressure: input module receives input signals and preclude the collection, there are two types of input signals ; continuously changing analog and the other is provided by a potentiometer, thermocouple, tachometer generator, various transmitter input / output signal voltage is generally higher, such as DC $24 \mathrm{~V}$ and AC $220 \mathrm{~V}$. Introduced from external interference and noise and sharp voltage may damage the CPU module components, or the right to use the programmable controller is not working properly. In the I / O 
modules, a photoelectric said combiner, optical controlled baby, small devices such as relays to isolate the external input circuit and the load, in addition to transmitting signals, level shifting and isolation as well as the role of $\mathrm{I} / \mathrm{O}$ modules.

In addition to the programmer used to enter and edit user program, but also can be used to monitor the operation of the PLC programming elements variety of working conditions. Programmers may be permanently connected to the programmable controller, the programmer will be taken down after the system can also run. Programmers generally used only when the program logic inputs, commissioning and maintenance, a programmer for multiple programmable controllers public.

Programmable controller uses $220 \mathrm{~V}$ AC or $24 \mathrm{~V}$ DC power supply. PLC internal DC power supply for the circuitry of each module, some of the programmable controller can provide $24 \mathrm{~V}$ DC power input circuit and external electronic detection device (such as a proximity switch), are driving the actuator DC power supply generally provided by the user.

Touch screen display and a touch Jian Guan is integrated programmable terminal, referred to as PT, it is a new generation of high-tech man-machine interface products. Designed for PLC applications designed touchscreen set host, input and output devices in one, suitable for use in harsh industrial environments (protection class IP65), use it as a man-machine interface, interactive, high reliability, simple programming as well as the advantages of easy connection with the PLC.

PLC work process can be divided into three phases, as follows: before the program is executed, the input terminal of the programmable controller ON / OFF status register reads the input image. In the execution of the program, even if the input status changes, the contents of the input image register is not changed. Until the input of the next processing phase of the scan cycle to read into this change. In addition, input from the through contact (ON) -> OFF (OFF) or from the off (OFF) -> pass $(\mathrm{ON})$ is determined to change state only, enter the filter there is a response delay time (about 10ms).

Instructions to the user program stored in the memory is read out from the ON / OFF state of the input image, registers and other devices in the image of the relevant register of the device, in order from the step 0 start operation, each time the results are written about the image register, Therefore, the contents of each device (except $\mathrm{X}$ ) of the image register as the program executes changing. Output relay contacts inside the action is determined by the content of the output image register.

All instruction is executed, the output image register on / off state of the output latch register transfer, become the actual output of the PLC. External PLC output contacts on the output device operation has a response time that have a delayed action.

\section{PLC Control System Design Program}

Program is divided into two blocks, namely manual and automatic two parts. When the mode selection button input point 2.01 of $0 \mathrm{~N}$, which often Jian contacts connected to perform a manual start procedure; when the mode selection button input point 2.01 is OFF, the normally closed contact closure and begin the automatic program, as the automatic mode which one, it depends on the mode button to select the semi-automatic or fully automatic. Adoption provided by OMRON CX-Prograinmer7.0 programming software programming.

Since the manual and automatic mode conversion by the JMP / JME instruction realized that, when executed by the condition JMP ON to OFF, the state of each output between JMP and JME remain unchanged. So in manual mode and automatic mode switching, generally reset operation to avoid misuse. During automatic operation To stop, press the stop button once W200 3.03 pairs of channel reset. In automatic operation process, if the stop button is pressed directly press the auto / manual mode selection button, W200 channel state will remain. When the manual operation is complete and then go to automatic mode, the original state W200 channel will cause malfunctions. In order to prevent this from happening, in the manual reset control program has taken measures. Since the W200 channel is reset, it will not appear when you switch malfunction. 


\section{Conclusion}

In this paper, a more complete design of a four DOF hydraulic driven mechanical hand control system is universal and practical. Program under the control of a programmable controller (PLC) and touch screen to achieve the material transport from one station to the next station, capable of accurate positioning and reliable completion of the action. In the course of their work by touching the screen (PT) real-time display the operating status of the robot, and the read-out velocity, displacement and pressure values robot, so you can easily control the manipulator. Completed graduate design process of the robot in the industrial field use with a more comprehensive understanding; familiar with the hydraulic system used in the control of; in the application of knowledge of PLC basic working principle, familiar with the module's designed to PLC as the core control system. By writing the commutation control circuit of the ladder program and touch screen program can automatically capture the movement of the hydraulic system and automatic control signals.

\section{References}

[1] Khong-Meng Tham, Krishnaswamy Nagaraj. A Low Supply Voltage High PSRR Voltage Reference in CMO Process [J]. IEEE Journal of Solid-state Circuits, 1995, 30

[2] Yu Fusheng, Duan Tangjia, Du Guilin. based on S7-200 PLC and other four degrees of freedom manipulator control system design [J]. hydraulic and pneumatic, 2013 (08): 89-91.

[3] Peng Yihang. Based on Siemens PLC technology robot control system design [J]. Value Engineering, 2014 (21): 51-52.

[4] Zheng Yulong, Zhang Yulian. PLC-based offshore drilling platform to the drill pipe conveyor robot control system design [J]. mechanical engineering design and manufacturing, 2014, (12): 51-54.

[5] Yang Rongqing. PLC-based pneumatic manipulator system status and control programming algorithm [J]. Architectural Engineering Technology and Design, 2015 (19): 1820-1820. 